Bull. Austral. Math. Soc.

VOL. 52 (1995) [231-234]

\title{
SIMPLE MODULES OVER THE COORDINATE RING OF QUANTUM AFFINE SPACE
}

\author{
KangJu Min and Sei-Qwon OH
}

The simple modules of $\mathcal{O}_{q}\left(\mathbb{C}^{n}\right)$, the coordinate ring of quantum affine space, are classified in the case when $q$ is a root of unity.

The coordinate ring of quantum affine space, $\mathcal{O}_{q}\left(\mathbb{C}^{n}\right)$, is the algebra generated by $x_{1}, \cdots, x_{n}$ satisfing the relations $x_{j} x_{i}=q x_{i} x_{j}, i<j, 0 \neq q \in \mathbb{C}$. Thus $\mathcal{O}_{q}\left(\mathbb{C}^{n}\right)$ is the iterated skew polynomial ring $\mathbb{C}\left[x_{1}\right]\left[x_{2} ; \tau_{2}\right] \cdots\left[x_{n} ; \tau_{n}\right]$, where automorphisms $\tau_{k}$ are defined by $\tau_{k}\left(x_{i}\right)=q x_{i}, i<k$. Therefore it is a Noetherian domain of Gelfand-Kirillov dimension $n$, and has a $\mathbb{C}$-basis given by the monomials $X^{I}$ where $I=\left(i_{1}, \cdots, i_{n}\right)$ is a multi-index with each $i_{j} \geqslant 0$.

The quantum matrices $\mathcal{O}_{q} M_{n}(\mathbb{C})$ and the quantised universal enveloping algebra $U_{q}(\mathfrak{s l}(n, \mathbb{C}))$ act on $\mathcal{O}_{q}\left(\mathbb{C}^{n}\right)$. The reader is referred to the articles [1] and [5] for further background and actions on $\mathcal{O}_{q}\left(\mathbb{C}^{n}\right)$.

The prime ideals and the primitive ideals of $\mathcal{O}_{q}\left(\mathbb{C}^{n}\right)$ are classified in [4] and [5, Section 3], in the case when $q$ is not a root of unity. In this note, we prove that there is a surjective map $\Psi$ from $\mathbb{C}^{n}$ onto the set of all the simple modules of $\mathcal{O}_{q}\left(\mathbb{C}^{n}\right)$, in the case when $q$ is a primitive $m$-th root of unity, such that

$$
\operatorname{dim}_{\mathbb{C}} \Psi(\underline{\alpha})=m^{[p / 2]},
$$

where $p$ is the number of nonzero $\alpha_{i}$ in $\underline{\alpha}=\left(\alpha_{1}, \cdots, \alpha_{n}\right)$ and $[x]$ denotes the greatest integer $\leqslant x$. Henceforce, assume throughout that $q$ is a primitive $m$-th root of unity unless stated otherwise.

PROPOSITION 1. Let $R$ be an algebra over a field $k, Z$ a finitely generated subalgebra contained in the center of $R$ and let $R$ be finitely generated as a $Z$-module. For a simple right $R$-module $M$, the following hold.
(i) $R$ is Noetherian.
(ii) $\operatorname{dim}_{k}(M)$ is finite.
(iii) $\operatorname{ann}_{R}(M)$ is a maximal ideal of $R$.
(iv) ann $R(M) \cap Z$ is a maximal ideal of $Z$.

Received 14th November, 1994

This research was partially supported by KOSEF and by BSRI-94-1427, Korea.

Copyright Clearance Centre, Inc. Serial-fee code: 0004-9729/95 \$A2.00+0.00. 
Proof: (i) Since $Z$ is Noetherian by Hilbert's basis theorem and $R$ is finitely generated as a $Z$-module, $R$ is also Noetherian.

(ii) $[3,9.5 .5$ (ii)].

(iii) and (iv) Look at the following monomorphisms:

$$
Z /\left(\operatorname{ann}_{R}(M) \cap Z\right) \stackrel{\alpha}{\longrightarrow} R / \operatorname{ann}_{R}(M), \quad R / \operatorname{ann}_{R}(M) \stackrel{\beta}{\longrightarrow} \operatorname{End}_{k}(M)
$$

where $\alpha$ is induced from the inclusion map from $Z$ into $R$ and $\beta$ is induced from right module structure map on $M$. Since $\operatorname{End}_{k}(M)$ is finite dimensional by (ii), $R / \operatorname{ann}_{R}(M)$ is Artinian and prime. Hence it is simple. Moreover, $Z /\left(\operatorname{ann}_{R}(M) \cap Z\right)$ is integral domain and Artinian. Therefore, $\operatorname{ann}_{R}(M) \cap Z$ and $\operatorname{ann}_{R}(M)$ are maximal ideals of $Z$ and $R$, respectively.

Corollary 2. Every simple right $\mathcal{O}_{q}\left(\mathbb{C}^{n}\right)$-module is finite dimensional.

Proof: The subalgebra $Z$ of $\mathcal{O}_{q}\left(\mathbb{C}^{n}\right)$ generated by $x_{i}^{m}, i=1, \cdots, n$, is contained in the center of $\mathcal{O}_{q}\left(\mathbb{C}^{n}\right)$ and $\mathcal{O}_{q}\left(\mathbb{C}^{n}\right)$ is finitely generated as a $Z$-module. This completes the proof by Proposition 1 (ii).

For convenience, set $i^{\prime}=n+1-i$ for $1 \leqslant i<(n+1) / 2$. If $n=2 k+1$ or $n=2 k$ then, for nonzero $\alpha_{1}, \cdots, \alpha_{n} \in \mathbb{C}$, let $M(\underline{\alpha})=M\left(\alpha_{1}, \cdots, \alpha_{n}\right)$ be the $\mathbb{C}$-vector space with basis $e\left(a_{1}, \cdots, a_{k}\right), 0 \leqslant a_{i} \leqslant m-1$. Then $M(\underline{\alpha})$ has a right $\mathcal{O}_{q}\left(\mathbb{C}^{n}\right)$-module structure defined as follows :

Case 1. $n=2 k+1$ :

$$
\begin{gathered}
e\left(a_{1}, \cdots, a_{k}\right) x_{i}=\alpha_{i} q^{-\left(a_{1}+\cdots+a_{i-1}\right)} e\left(a_{1}, \cdots, a_{i-1}, a_{i}+1, a_{i+1}, \cdots, a_{k}\right), \\
1 \leqslant i \leqslant k \\
e\left(a_{1}, \cdots, a_{k}\right) x_{i^{\prime}}=\alpha_{i}^{-1} \alpha_{i^{\prime}} q^{-\left(a_{1}+\cdots+a_{i}\right)+1} e\left(a_{1}, \cdots, a_{i-1}, a_{i}+(-1), a_{i+1}, \cdots, a_{k}\right), \\
1 \leqslant i \leqslant k \\
e\left(a_{1}, \cdots, a_{k}\right) x_{k+1}=\alpha_{k+1} q^{-\left(a_{1}+\cdots+a_{k}\right)} e\left(a_{1}, \cdots, a_{k}\right)
\end{gathered}
$$

Case 2. $n=2 k$ :

$$
\begin{gathered}
e\left(a_{1}, \cdots, a_{k}\right) x_{i}=\alpha_{i} q^{-\left(a_{1}+\cdots+a_{i-1}\right)} e\left(a_{1}, \cdots, a_{i-1}, a_{i}+1, a_{i+1}, \cdots, a_{k}\right), \\
1 \leqslant i \leqslant k \\
e\left(a_{1}, \cdots, a_{k}\right) x_{i^{\prime}}=\alpha_{i}^{-1} \alpha_{i^{\prime}} q^{-\left(a_{1}+\cdots+a_{i}\right)+1} e\left(a_{1}, \cdots, a_{i-1}, a_{i}+(-1), a_{i+1}, \cdots, a_{k}\right), \\
1 \leqslant i \leqslant k-1 \\
e\left(a_{1}, \cdots, a_{k}\right) x_{k+1}=\alpha_{k+1} q^{-\left(a_{1}+\cdots+a_{k}\right)} e\left(a_{1}, \cdots, a_{k}\right) ;
\end{gathered}
$$

where $\dot{+}$ is addition in the additive group $\mathbb{Z}_{m}$. To confirm the well-definedness of these rules, it suffices to check that

$$
e\left(a_{1}, \cdots, a_{k}\right) x_{j} x_{i}=q e\left(a_{1}, \cdots, a_{k}\right) x_{i} x_{j}, 1 \leqslant i<j \leqslant n .
$$


These are all routinely verified.

Proposition 3. The right $\mathcal{O}_{q}\left(\mathbb{C}^{n}\right)$-module $M(\underline{\alpha})$ is simple.

Proof: If $n=2 k+1$ and $e\left(a_{1}, \cdots, a_{k}\right) \neq e\left(a_{1}^{\prime}, \cdots, a_{k}^{\prime}\right)$, choose an index $i$ such that $a_{1}=a_{1}^{\prime}, \cdots, a_{i-1}=a_{i-1}^{\prime}, a_{i} \neq a_{i}^{\prime}$. The vectors $e\left(a_{1}, \cdots, a_{k}\right)$ and $e\left(a_{1}^{\prime}, \cdots, a_{k}^{\prime}\right)$ are eigenvectors of $x_{i} x_{i^{\prime}}$ associated with the distinct eigenvalues $\alpha_{i^{\prime}} q^{-2\left(a_{1}+\cdots+a_{i-1}\right)-a_{i}}$ and $\alpha_{i^{\prime}} q^{-2\left(a_{1}^{\prime}+\cdots+a_{i-1}^{\prime}\right)-a_{i}^{\prime}}$, respectively.

If $n=2 k$, let $e\left(a_{1}, \cdots, a_{k}\right), e\left(a_{1}^{\prime}, \cdots, a_{k}^{\prime}\right)$ and $i$ be as in the case $n=2 k+1$. If $i<k$ then $e\left(a_{1}, \cdots, a_{k}\right)$ and $e\left(a_{1}^{\prime}, \cdots, a_{k}^{\prime}\right)$ are eigenvectors of $x_{i} x_{i^{\prime}}$ associated with the distinct eigenvalues $\alpha_{i^{\prime}} q^{-2\left(a_{1}+\cdots+a_{i-1}\right)-a_{i}}$ and $\alpha_{i^{\prime}} q^{-2\left(a_{1}^{\prime}+\cdots+a_{i-1}^{\prime}\right)-a_{i}^{\prime}}$, respectively, and if $i=k$ then $e\left(a_{1}, \cdots, a_{k}\right)$ and $e\left(a_{1}^{\prime}, \cdots, a_{k}^{\prime}\right)$ are eigenvectors of $x_{k+1}$ associated with the distinct eigenvalues $\alpha_{k+1} q^{-\left(a_{1}+\cdots+a_{k}\right)}$ and $\alpha_{i^{\prime}} q^{-\left(a_{1}^{\prime}+\cdots+a_{k}^{\prime}\right)}$, respectively.

Hence every nonzero submodule of $M(\underline{\alpha})$ contains a vector $e\left(a_{1}, \cdots, a_{k}\right)$, thus $M(\underline{\alpha})$ is simple by the action of $x_{i}, 1 \leqslant i \leqslant k$.

Proposition 4. Let a simple right $\mathcal{O}_{q}\left(\mathbb{C}^{n}\right)$-module $N$ be $x_{i}$-torsion free for each $i=0, \cdots, n$. Then $N$ is isomorphic to $M(\underline{\alpha})$ for some $\underline{\alpha}=\left(\alpha_{i}\right) \in\left(\mathbb{C}^{*}\right)^{n}$.

Proof: Let $n=2 k+1$. Since each $x_{i}^{m}, x_{i} x_{i^{\prime}}, i=1, \cdots, k$ and $x_{k+1}$ commutes and $N$ is finite dimensional, there is a common eigenvector $v$ of $x_{i}^{m}, x_{i} x_{i^{\prime}}, i=1, \cdots, k$ and $x_{k+1}$. Put $v x_{i}^{m}=\nu_{i} v, v x_{i} x_{i^{\prime}}=\alpha_{i^{\prime}} v, v x_{k+1}=\alpha_{k+1} v, i=1, \cdots, k$. For each $i=1, \cdots, k$, let $\alpha_{i}$ be an $m$-th root of $\nu_{i}$. Notice that the $\alpha_{i}$ are all nonzero and

$$
v x_{k}^{a_{k}} \cdots x_{1}^{a_{1}} x_{i^{\prime}}= \begin{cases}\alpha_{i^{\prime}} q^{-\left(a_{1}+\cdots+a_{i}\right)+1} v x_{k}^{a_{k}} \cdots x_{i}^{a_{i}-1} \cdots x_{1}^{a_{1}}, & a_{i}>0 \\ \nu_{i}^{-1} \alpha_{i^{\prime}} q^{-\left(a_{1}+\cdots+a_{i}\right)+1} v x_{k}^{a_{k}} \cdots x_{i}^{m-1} \cdots x_{1}^{a_{1}}, & a_{i}=0 .\end{cases}
$$

Define a linear transformation

$$
\psi: M(\underline{\alpha}) \longrightarrow N, \psi\left(e\left(a_{1}, \cdots, a_{k}\right)\right)=\alpha_{1}^{-a_{1}} \cdots \alpha_{k}^{-a_{k}} v x_{k}^{a_{k}} \cdots x_{1}^{a_{1}} .
$$

It is routinely verified that

$$
\psi\left(e\left(a_{1}, \cdots, a_{k}\right) x_{i}\right)=\psi\left(e\left(a_{1}, \cdots, a_{k}\right)\right) x_{i}, \quad i=1, \cdots, n .
$$

Hence $\psi$ is an $\mathcal{O}_{q}\left(\mathbb{C}^{n}\right)$-homomorphism, and thus it is an isomorphism because $M(\underline{\alpha})$ and $N$ are both simple. The proof of the case when $n=2 k$ is similar.

THEOREM 5. There is a surjective map $\Psi$ from $\mathbb{C}^{n}$ onto the set of all the simple right $\mathcal{O}_{q}\left(\mathbb{C}^{n}\right)$-modules such that $\operatorname{dim}_{\mathbb{C}} \Psi(\underline{\alpha})=m^{[p / 2]}$, where $p$ is the number of nonzero $\alpha_{i}$ in $\underline{\alpha}=\left(\alpha_{i}\right)$ and $[x]$ is the greatest integer $\leqslant x$.

Proof: Let $M$ be a simple $\mathcal{O}_{q}\left(\mathbb{C}^{n}\right)$-module and let $Z$ be the subalgebra generated by $x_{i}^{m}, i=1, \cdots, n$. Then $\operatorname{ann}(M) \cap Z$ is a maximal ideal of $Z$ by Proposition 1 (iv). 
Hence $x_{i}^{m}-\lambda_{i} \in \operatorname{ann}(M)$ for some $\lambda_{i} \in \mathbb{C}, i=1, \cdots, n$. If $\lambda_{i}=0$ then $x_{i} \in$ ann $(M)$ since $x_{i}$ is a normal element of $\mathcal{O}_{q}\left(\mathbb{C}^{n}\right)$ and $\operatorname{ann}(M)$ is prime. For convenience, assume that $\lambda_{1}, \cdots, \lambda_{p}$ are all nonzero and $\lambda_{p+1}=\cdots=\lambda_{n}=0$. Thus $M$ is a simple $\mathcal{O}_{q}\left(\mathbb{C}^{p}\right)-$ module and is $x_{i}$-torsion free for each $i=1, \cdots, p$, since ann $(M)$ contains $x_{p+1}, \cdots, x_{n}$ and $\mathcal{O}_{q}\left(\mathbb{C}^{n}\right) /\left\langle x_{p+1}, \cdots, x_{n}\right\rangle$ is isomorphic to $\mathcal{O}_{q}\left(\mathbb{C}^{p}\right)$. Hence the result follows from Proposition 4.

REMARK 1. The map $\Psi$ of Theorem 5 is not injective.

Proof: Let $n=2 k+1$ and $\underline{\alpha}, \underline{\beta}$ be two elements in $\left(\mathbb{C}^{*}\right)^{n}$ such that $\alpha_{i}=\beta_{i}, i \neq$ $k+1, k+2$ and $\alpha_{k+1}=q^{-1} \beta_{k+1}, \alpha_{k+2}=q^{-1} \beta_{k+2}$. Then, it is easy to see that the $\operatorname{map} \psi: M(\underline{\alpha}) \longrightarrow M(\underline{\beta})$ given by $\psi\left(e\left(a_{1}, \cdots, a_{k}\right)\right)=e\left(a_{1}, \cdots, a_{k-1}, a_{k}+1\right)$ is an isomorphism.

REMARK 2. All primitive ideals of $\mathcal{O}_{q}\left(\mathbb{C}^{n}\right)$ are annihilators of $\Psi(\underline{\alpha}), \underline{\alpha} \in \mathbb{C}^{n}$.

REMARK 3. If $q$ is not a root of unity then every finite dimensional simple $\mathcal{O}_{q}\left(\mathbb{C}^{n}\right)$ module is one-dimensional by $[2,1.3]$. The classification of the one-dimensional simple modules is a fairly easy exercise.

REMARK 4. Smith classified all simple modules of $\mathcal{O}_{q}\left(\mathbb{C}^{2}\right)$ in $[6$, pp. 123].

\section{REFERENCES}

[1] L.D. Fadeev, N.Y. Reshetikhin and L.A. Takhtadzhyan, 'Quantization of Lie groups and Lie algebras', Leningrad Math. J. 1 (1990), 193-225.

[2] K.R. Goodearl and E.S. Letzter, 'Prime factor algebras of the coordinate ring of quantum matrices', Proc. Amer. Math. Soc. 121 (1994), 1017-1025.

[3] J.C. McConnell and J.C. Robson, Noncommutative Noetherian rings, Pure and applied mathematics (John Wiley and Sons, 1987).

[4] Sei-Qwon Oh, Primitive ideals in algebras of functions on certain quantum spaces, Doctoral dissertation (University of Cincinnati, 1992).

[5] S.P. Smith, 'Quantum groups: An introduction and survey for ring theorists', in Noncommutative rings, (S. Montgomery and L. Small, Editors), Math. Sci. Res. Inst. Publ. 24 (Springer-Verlag, New York, 1992), pp. 131- 178.

[6] S.P. Smith, Noncommutative algebras, Lecture Notes (University of Washington, 1990).

\footnotetext{
Department of Mathematics

Chungnam National University

Taejon 305-764

Korea
} 